\title{
INVESTIGATION OF THE CARBOHYDRATES OF CAMELINA SATIVA (L.) CRANTZ AND CAMELINA MICROCARPA ANDRZ.
}

\author{
Tetiana Tsykalo, Serhiy Trzhetsynskyi
}

The aim of the work was to study the monosaccharide composition of WSPS, PS and HC, isolated from the raw materials of Camelina sativa and Camelina microcarpa and to establish the quantitative content of these fractions.

Materials and methods. The analysis of the composition of biologically active substances of carbohydrate nature was carried out in herb and seeds of Camelina sativa (variety "Slavutych") and Camelina microcarpa. Samples of seeds for growing plants were provided by the National Center for Genetic Resources of Plants of Ukraine (The Plant Production Institute ND. V.YA. YURIEVA, NAAS of Ukraine).

For the studies, the carbohydrates were separated by the Bailey method into monosaccharide fractions, which were used for TLC and quantification using a modified Dreywood spectrophotometric method with anthrone reagent in concentrated sulfuric acid.

Results. The presence of glucose, galactose and arabinose was determined in the hydrolysates of polysaccharide fractions of Camelina sativa of herb and seeds. Galactose, glucose, arabinose and xylose were found in the hydrolysates of polysaccharide fractions of Camelina microcarpa herb and seeds. The highest content of WSPS was determined in the herb of Camelina sativa, and the lowest - in the seeds of Camelina microcarpa. PS in the largest number were also found in the herb, and in the smallest - in the seeds of Camelina sativa. The content of the amount of HC was the highest in the herb of Camelina sativa, and the smallest - in the seeds of Camelina sativa.

Conclusions. The presence of 3 monosaccharides in the raw material of Camelina sativa and 4 monosaccharides in the raw material of Camelina microcarpa was established by TLC. The quantitative content of monosaccharide fractions was determined by the spectrophotometric method, which in total predominated in Camelina sativa

Keywords: carbohydrates, WSPS, PS, HC, Camelina sativa (L.) Crantz, Camelina microcarpa Andrz., TLC, spectrophotometric research

How to cite:

Tsykalo, T., Trzhetsynskyi, S. (2021). Investigation of the carbohydrates of Camelina sativa (L.) Crantz and Camelina microcarpa Andrz. ScienceRise: Pharmaceutical Science, 2 (30), 13-16. doi: http://doi.org/10.15587/2519-4852.2021.230045

(C) The Author(s) 2021

This is an open access article under the Creative Commons CC BY license

\section{Introduction}

Polysaccharides are one of the most important members of the family of biopolymers and attract the attention of scientists in various fields. Polysaccharides (glycans) are high molecular weight carbohydrates consisting of monosaccharides linked by glycosidic bonds in linear or branched chains. The degree of polymerization of polysaccharides is from 10-20 to several thousand residues, may have $\alpha$ - or $\beta$-configuration of the glycosidic center $[1,2]$.

Carbohydrates are the main part of the plant body, which accounts for most of the dry mass of plants, providing the skeletal functions of the body and cells cellulose, hemicellulose and pectin [3]. Another important function of polysaccharides is to provide energy to plant organisms, turning into monosaccharides [4].

Polysaccharides consist of the following fractions: water-soluble polysaccharides (WSPS), pectin substances (PS) and hemicellulose (HC) [5].

Pectins are highly heterogeneous polysaccharides, which are traditionally characterized by the fact that they are relatively easily extracted with hot acid or chelators and contain many galacturonic acid residues [5]. Pectin polysaccharides have great potential in the healthcare, food and cosmetics industries [6].

Studies demonstrate that pectin polysaccharides are physiologically active substances with immunomodulating properties, including anti-inflammatory activity, they lower cholesterol and triglyceride in the blood serum, normalize glucose metabolism, bind and remove toxins and radionuclides from the body, regulate work and provide protection of the gastrointestinal tract, have anticarcinogenic and antimetastatic effects [7].

Hemicelluloses are types of plant cell wall polysaccharides, and the world's second most abundant renewable polymers after cellulose in lignocellulosic materials. Hemicelluloses traditionally contain pentoses and hexoses, among the monosaccharides predominate: xylans, mannans and galactans. These polysaccharides differ greatly from each other in structure and physicochemical properties. Hemicellulose can be extracted with alkalis [5].

Xylan, the major hemicellulosic component of lignocellulose and the second most abundant polysaccharide after cellulose [8]. Xylan has immunomodulatory effects, and has been combined with chitosan to form a 
composite hydrogel to improve the healing of bone fractures [9].

Carbohydrates are an important class of natural compounds with a diverse spectrum of biological effects on the human body. It is established that plant polysaccharides, which were previously considered inert substances, show a wide range of biological activity: reparative [10], hypoglycemic [11], emollient, participate in the creation of immunity, increase the body's resistance [12], potentiate the pharmacological activity of flavonoids and other biologically active substances, have a mucolytic effect [13]. Plant polysaccharides also have antitumor [14], anti-radiation and antiviral effects $[15,16]$. Pentasaccharides may have anticoagulant action [17]. Sulphated polysaccharides have a wide range of important bioactivities, including antioxidant [18], antithrombotic activity. According to the literature, it is known that most plant polysaccharides are relatively nontoxic and do not cause significant side effects [19].

Little-studied plants with experience in folk medicine can become a new source of carbohydrates. Such plants include members of the family Brassicaceae, genus Camelina (L.) Crantz - Camelina sativa (L.) Crantz and Camelina microcarpa Andrz., which are common in Ukraine and have long been used as hypoglycemic, hypolipidemic, antioxidant agents [20].

The aim of the work was to study the monosaccharide composition of WSPS, PS and HC, isolated from the raw materials of Camelina sativa and Camelina microcarpa and to establish the quantitative content of these fractions.

\section{Research planning (methodology)}

The analysis of the scientific literature showed that the chemical composition of the raw materials of $\mathrm{C}$. sativa and C. microcarpa has not been sufficiently studied. There is little information in the available reliable sources on the carbohydrate composition of C. sativa. No information was found on the composition of carbohydrates in the raw materials of C. microcarpa.

The study of the carbohydrate composition of the plant is a promising area, as this class of compounds has a variety of pharmacological properties.

Based on this, we aimed to study the monosaccharide composition of WSPS, PS and HC by thin layer chromatography and spectrophotometric method, as these methods are among the most optimal and available for analysis of these objects.

To achieve this goal, the first stage was the selection of WSPS from raw materials of species of the genus Camelina. The second stage was to obtain PS from the meal left after the separation of WSPS. The third stage involved obtaining $\mathrm{HC}$ and $\mathrm{HC}$ A from the meal left after the separation of the PS. The fourth stage was the establishment of the monosaccharide composition of WSPS, PS and HC by TLC and determination of the quantitative content of these fractions by spectrophotometric method.

\section{Materials and methods}

Analysis of the composition of biologically active substances of carbohydrate nature was carried out in herb and seeds of $C$. sativa (variety "Slavutych") and C. microcarpa. Raw material was grown and harvested in the territory of Zaporizhzhia region (Ukraine) in the summer of 2018-2019. Herb was harvested at the start of the flowering stage. Seeds were harvested when $60-70 \%$ of the ripe fruits were ripe to prevent mass rash. Samples of seeds for cultivation were provided by the National Center for Plant Genetic Resources of Ukraine (The Plant Production Institute named after V. Y. Yuriev, NAAS of Ukraine, Kharkiv). The raw material was dried in the shade in the open air, stirring periodically.

Preliminary identification of carbohydrates was performed using known qualitative reactions (precipitation with $96 \%$ ethanol, Fehling's reagent, carbazole solution and sulfuric acid) in an aqueous extract, which was prepared in a ratio of raw material-extractant 1:50 in a water bath for 1 hour [21].

For further studies, the carbohydrates were separated into monosaccharide fractions by the Bailey method, which were used for TLC and quantification using a modified Dreywood spectrophotometric method with anthron-sulfur reagent [22].

For statistical accuracy, the experiments were performed at least five times.

To determine the monomer composition of WSPS, PS and HC by chromatographic method, acid hydrolysis of fractions was previously performed. To do this, a portion of the polysaccharide fractions (about $0.5 \mathrm{~g}$ ) was dissolved in $2 \mathrm{ml}$ of purified water and hydrolyzed with $2 \mathrm{ml}$ of a $20 \%$ solution of sulfuric acid while heating in a water bath under reflux for 5 hours. The hydrolysates were neutralized to a neutral reaction according to the universal indicator of barium carbonate. The resulting solutions were filtered, then the filtrates were evaporated to $0.5 \mathrm{ml}$ and applied to a plate of Sorbfil plates for chromatography in the system acetonitrile-water $(85: 15)$. Standard samples of monosaccharides (Sigma-Aldrich) were applied side by side. Chromatograms after drying in air were treated with an aniline phthalate reagent and heated in an oven at a temperature of $100-105^{\circ} \mathrm{C}$.

\section{Results}

Preliminary identification of carbohydrates was performed using qualitative reactions. The monomeric composition of the polysaccharide fractions was determined by TLC. The results of TLC are presented in Table 1.

The presence of galactose $(\mathrm{Rf}=0.19 \pm 0.004)$, glucose $(\mathrm{Rf}=0.22 \pm 0.005)$ and arabinose $(\mathrm{Rf}=0.28 \pm 0.005)$ was determined in the hydrolysates of polysaccharide fractions of C. sativa herb. In seeds of C. sativa - galactose $(\mathrm{Rf}=0.19 \pm 0.004)$, glucose $(\mathrm{Rf}=0.21 \pm 0.004)$ and arabinose $(\mathrm{Rf}=0.27 \pm 0.005)$.

Galactose $\quad(\mathrm{Rf}=0.19 \pm 0.003)$, glucose $\quad(\mathrm{Rf}=0.21 \pm$ $\pm 0.004)$, arabinose $(\mathrm{Rf}=0.27 \pm 0.003)$ and xylose $(\mathrm{Rf}=0.30 \pm$ $\pm 0.004)$ were found in the hydrolysates of polysaccharide fractions of C. microcarpa herb. In C. microcarpa seeds galactose $(\mathrm{Rf}=0.18 \pm 0.002)$, glucose $(\mathrm{Rf}==0.20 \pm 0.003)$, arabinose $(\mathrm{Rf}=0.28 \pm 0.005)$ and xylose $(\mathrm{Rf}=0.31 \pm 0.004)$.

Quantitative content of polysaccharide fractions was performed spectrophotometrically with anthrone reagent in terms of arabinose and galactose. The results of the study are presented in Table 2. 
The results of studying the monomeric composition of polysaccharide fractions of raw materials of the genus Camelina by TLC

\begin{tabular}{|c|c|c|c|c|c|}
\hline Object of study & Hydrolyzate of fractions & Galactose & Glucose & Arabinosis & Xylose \\
\hline \multirow{3}{*}{ C. sativa herb } & WSPS & - & + & + & - \\
\hline & PS & + & - & - & - \\
\hline & $\mathrm{HC}$ & + & - & + & - \\
\hline \multirow{3}{*}{ C. sativa seed } & WSPS & - & - & + & - \\
\hline & PS & + & + & - & - \\
\hline & $\mathrm{HC}$ & + & - & + & - \\
\hline \multirow{3}{*}{ C. microcarpa herb } & WSPS & - & + & + & - \\
\hline & PS & + & - & + & - \\
\hline & $\mathrm{HC}$ & + & - & - & + \\
\hline \multirow{3}{*}{ C. microcarpa seed } & WSPS & - & + & + & - \\
\hline & PS & + & - & + & - \\
\hline & $\mathrm{HC}$ & + & - & + & + \\
\hline
\end{tabular}

Table 2

Quantitative content of polysaccharide fractions in raw materials of red species

\begin{tabular}{|l|c|c|c|c|c|}
\hline \multirow{2}{*}{ Object of study } & \multicolumn{5}{|c|}{ Quantitative content of polysaccharide fractions, \% } \\
\cline { 2 - 6 } & WSPS & PS & HC & HC A & HC B \\
\hline C. sativa herb & $3.64 \pm 0.07$ & $2.99 \pm 0.14$ & $4.83 \pm 0.17$ & $0.53 \pm 0.04$ & $4.32 \pm 0.21$ \\
\hline C. sativa seed & $1.59 \pm 0.04$ & $0.45 \pm 0.03$ & $1.79 \pm 0.09$ & $0.59 \pm 0.07$ & $1.21 \pm 0.15$ \\
\hline C. microcarpa herb & $2.24 \pm 0.06$ & $2.52 \pm 0.06$ & $3.36 \pm 0.26$ & $1.03 \pm 0.19$ & $2.33 \pm 0.23$ \\
\hline C. microcarpa seed & $1.12 \pm 0.03$ & $0.57 \pm 0.01$ & $2.15 \pm 0.17$ & $0.34 \pm 0.02$ & $1.81 \pm 0.19$ \\
\hline
\end{tabular}

As can be seen from the data given in the table, among the polysaccharide fractions of raw materials of both types, HC predominates, namely HC B. According to the total content of all fractions, is dominated herb by C. sativa. The lowest total content of all fractions in the seeds of C. microcarpa. The total content of polysaccharide fractions of $\mathrm{C}$. sativa is three times higher than the content of this fractions in the seeds of this plant. At the same time, the total content of polysaccharide fractions in the C. microcarpa is twice as high as in the seeds of this plant.

\section{Discussion}

The presence of glucose, galactose and arabinose was determined in the hydrolysates of polysaccharide fractions of herb and seeds of C. sativa. Galactose, glucose, arabinose, and xylose were found in the hydrolysates of polysaccharide fractions of herb and seeds of $\mathrm{C}$. microcarpa.

According to the results of fractionation, it was found that the content of polysaccharides in the raw materials of both types of Camelina is quite similar. The highest content of water-soluble polysaccharides was determined in C. sativa herb, and the lowest - in C. microcarpa seeds. Pectic substances are also found in the largest quantities in $\mathrm{C}$. sativa herb. The smallest amount of pectin was contained in C. sativa seeds. The highest number of hemicelluloses was in C. sativa grass, and the lowest - in C. sativa seeds.

Our studies on the monosaccharide composition of C. sativa seeds coincide with studies [23], which de- termined the qualitative and quantitative content of galactose, glucose, rhamnose and xylose. By TLC, we found arabinose in the seeds, but did not detect xylose. It may depend on the variety and location of the plant. No studies of carbohydrates in Camelina sativa grass and in Camelina microcarpa raw materials are known.

Study limitations. The study requires additional investigation of monosaccharides by HPLC to confirm the presence of individual monosaccharide compounds and their quantification.

Prospects for further research. A significant number of monosaccharide fractions indicates the need for in-depth pharmacological studies of this raw material.

\section{Conclusions}

The presence of 3 monosaccharides in raw materials of C. sativa and 4 monosaccharides in raw materials of C. microcarpa was determined by TLC. The quantitative content of monosaccharide fractions was determined by the spectrophotometric method, which prevailed in C. sativa. The results of these studies expand the information on biologically active substances of C. sativa and C. microcarpa. Considering the results of phytochemical analysis, it can be assumed that polysaccharides of the genus Camelina are promising for further research to develop new effective and safe drugs for use in medical practice.

\section{Conflict of interests}

The authors declare that they have no conflicts of interest.

References

1. Yin, M., Zhang, Y., Li, H. (2019). Advances in Research on Immunoregulation of Macrophages by Plant Polysaccharides. Frontiers in Immunology, 10, 1-9. doi: http://doi.org/10.3389/fimmu.2019.00145 
2. Trigui, I., Yaich, H., Sila, A., Cheikh-Rouhou, S., Bougatef, A., Blecker, C. et. al. (2018). Physicochemical properties of water-soluble polysaccharides from black cumin seeds. International Journal of Biological Macromolecules, 117, 937-946. doi: http://doi.org/10.1016/j.ijbiomac.2018.05.202

3. Zorikova, O. V., Manyahin, A. Yu., Borovaya, S. A., Railko, S. P. (2018). Seasonal dynamics of polysaccharid content in raw materials reynoutria japonica. Chemistry of Plant Raw Material, 3, 33-39. doi: http://doi.org/10.14258/jcprm.2018033777

4. Kolisnyk, S., Khanin, V., Umarov, U., Koretnik, O. (2020). Study of the monosaccharide composition of water-soluble polysaccharide complexes and pectic substances of Pimpinella anisum herbs. ScienceRise: Pharmaceutical Science, 3 (25), $33-38$. doi: http://doi.org/10.15587/2519-4852.2020.206776

5. Scheller, H. V., Ulvskov, P. (2010). Hemicelluloses. Annual Review of Plant Biology, 61 (1), 263-289. doi: http://doi.org/10.1146/annurev-arplant-042809-112315

6. Minzanova, S., Mironov, V., Arkhipova, D., Khabibullina, A., Mironova, L., Zakirova, Y., Milyukov, V. (2018). Biological Activity and Pharmacological Application of Pectic Polysaccharides: A Review. Polymers, 10 (12), 1407. doi: http://doi.org/10.3390/polym10121407

7. Zaitseva, O., Khudyakov, A., Sergushkina, M., Solomina, O., Polezhaeva, T. (2020). Pectins as a universal medicine. Fitoterapia, 146, 104676. doi: http://doi.org/10.1016/j.fitote.2020.104676

8. Tokuda, G., Mikaelyan, A., Fukui, C., Matsuura, Y., Watanabe, H., Fujishima, M., Brune, A. (2018). Fiber-associated spirochetes are major agents of hemicellulose degradation in the hindgut of wood-feeding higher termites. Proceedings of the National Academy of Sciences, 115 (51), E11996-E12004. doi: http://doi.org/10.1073/pnas.1810550115

9. Bush, J. R., Liang, H., Dickinson, M., Botchwey, E. A. (2016). Xylan hemicellulose improves chitosan hydrogel for bone tissue regeneration. Polymers for Advanced Technologies, 27 (8), 1050-1055. doi: http://doi.org/10.1002/pat.3767

10. Liu, B., Wang, J.-L., Wang, X.-M., Zhang, C., Dai, J.-G., Huang, X.-M., Gao, J.-M. (2020). Reparative effects of lycium barbarum polysaccharide on mouse ovarian injuries induced by repeated superovulation. Theriogenology, 145, 115-125. doi: http://doi.org/10.1016/j.theriogenology.2020.01.048

11. Wang, L., Zhang, B., Xiao, J., Huang, Q., Li, C., Fu, X. (2018). Physicochemical, functional, and biological properties of water-soluble polysaccharides from Rosa roxburghii Tratt fruit. Food Chemistry, 249, $127-135$. doi: http://doi.org/10.1016/j.foodchem.2018.01.011

12. Nie, L., Xiao, Q., Liu, S., Li, B., Duan, J., Fan, Y., Zhu, H. (2019). Immune-enhancing effects of polysaccharides MLN-1 from by-product of Mai-luo-ning in vivo and in vitro. Food and Agricultural Immunology, 30 (1), 369-384. doi: http://doi.org/10.1080/09540105.2019.1582612

13. Guo, T., Qing Wei, J., Ping Ma, J. (2015). Antitussive and expectorant activities of Potentilla anserina. Pharmaceutical Biology, 54 (5), 807-811. doi: http://doi.org/10.3109/13880209.2015.1080734

14. Chen, L., Huang, G. (2018). Antitumor Activity of Polysaccharides: An Overview. Current Drug Targets, 19 (1), 89-96. doi: http://doi.org/10.2174/1389450118666170704143018

15. Shi, J., Cheng, C., Zhao, H., Jing, J., Gong, N., Lu, W. (2013). In vivo anti-radiation activities of the Ulva pertusa polysaccharides and polysaccharide-iron (III) complex. International Journal of Biological Macromolecules, 60, 341-346. doi: http://doi.org/10.1016/j.ijbiomac.2013.06.001

16. Chen, L., Huang, G. (2018). The antiviral activity of polysaccharides and their derivatives. International Journal of Biological Macromolecules, 115, 77-82. doi: http://doi.org/10.1016/j.ijbiomac.2018.04.056

17. Brandao, G. M., Junqueira, D. R., Rollo, H. A., Sobreira, M. L. (2017). Pentasaccharides for the treatment of deep vein thrombosis. Cochrane Database of Systematic Reviews. doi: http://doi.org/10.1002/14651858.cd011782.pub2

18. Chen, G., Kan, J. (2018). Characterization of a novel polysaccharide isolated from Rosa roxburghii Tratt fruit and assessment of its antioxidant in vitro and in vivo. International Journal of Biological Macromolecules, 107, 166-174. doi: http://doi.org/10.1016/j.ijbiomac.2017.08.160

19. Cho, C.-W., Song, Y.-R., Lim, W.-C., Hwang, Y.-H., Rhee, Y. K., Choi, J. W., Hong, H.-D. (2020). Acute Oral Toxicity and Genotoxicity of Polysaccharide Fraction from Young Barley Leaves (Hordeum vulgare L.). Foods, 9 (6), 809. doi: http://doi.org/10.3390/foods9060809

20. Shevchenko, I. A., Poliakov, O. I., Vedmedieva, K. V., Komarova, I. B. (2017). Strategy of production of oilseeds in Ukraine (rare crops). Zaporizhzhia: STATUS, 40.

21. Marchyshyn, S. M., Kudria, V. V., Dakhym, I. S., Zarichanska, O. V. (2018). Research of carbohydrates from great burnet (Sanguisorba officinalis L.) rhizomes with roots and herb. Medical and Clinical Chemistry, 1, 93-99. doi: http://doi.org/10.11603/mcch.2410-681x.2018.v0.i1.8885

22. Yushchyshena, O. V., Tsurkan, O. O., Korablyova, O. A., Kovalska, N. P. (2013). Investigation of essential oil of leaves, stems and inflorescences of vitex agnus-castus 1. and V. Cannabifolia sieb. Pharmaceutical Review, 4, 38-42.

23. Li, N., Qi, G., Sun, X. S., Wang, D. (2016). Characterization of gum isolated from Camelina seed. Industrial Crops and Products, 83, 268-274. doi: http://doi.org/10.1016/j.indcrop.2016.01.029

Received date 19.11.2020

Accepted date 21.04.2021

Published date 30.04.2021

Tetiana Tsykalo, Postgraduate Student, Department of Pharmacognosy, Pharmacology and Botany, Zaporizhzhia State Medical University, Maiakovskoho ave., 26, Zaporizhzhia, Ukraine, 69035

E-mail: tetyanatsykalo@ukr.net

Serhiy Trzhetsynskyi, Doctor of Biological Sciences, Professor, Head of Department, Department of Pharmacognosy, Pharmacology and Botany, Zaporizhzhia State Medical University, Maiakovskoho ave., 26, Zaporizhzhia, Ukraine, 69035

E-mail: sersh_dm@ukr.net 